\title{
Enhanced QoS by Service Differentiation in MAC-Layer for WLAN
}

\author{
Rathnakar Achary, \\ Alliance Business Academy \\ Bangalore, India \\ V. Vaityanathan, \\ Dept. of C.Sc. \& Engg. \\ SASTRA University \\ Tanjavur Tamil Nadu
}

\author{
Pethur Raj Chellaih \\ Wipro Technologies \\ Bangalore, India \\ Nagarajan $\mathrm{S}$ \\ School of Computing \\ SASTRA University \\ Tanjavur Tamil Nadu
}

\begin{abstract}
IEEE 802.11 wireless Local Area Network (WLAN) have become more and more popular due to easy to deployment and lower cost. It can only provide a best effort service and do not have quality of service (QoS) support for real time (RT) traffic. The IEEE Task Group has been proposed a new standard IEEE 802.11e[1] to address this MAC-layer QoS issue of WLAN. In this paper we proposed a new service differentiation mechanism for admission control in the MAC layer of WLAN for enhancing the QoS.
\end{abstract}

\section{Keywords}

Admission control, service differentiation, priority scheduling, guaranteed Service, Differentiated Service.

\section{INTRODUCTION}

WLANs are widely adopted worldwide due to its prevailing features for the companies and domestic users. Today the IEEE 802.11 WLAN standard family represents one of the most widely adopted technologies for delivering different traffics through wireless network. The diverse requirements of heterogeneous traffic contending the wireless channel heavily affect the efficient delivery over WLANs. To provide better QoS compared to the legacy 802.11, IEEE Task Group introduced IEEE $802.11 \mathrm{e}$ [1] by enhancing the MAC scheme, named hybrid coordination function (HCF) which operates with two access modes; a contention based enhanced distributed channel access (EDCA) and a contention free channel access scheme hybrid coordination function controlled channel access (HCCA). The EDCA scheme is based on carrier sense multiple accesses with collision avoidance (CSMA/CA). where collision avoidance is realized through a random backoff time for transmission if a station (STA) has a frame ready for delivery, it starts contending the channel once the STA successfully detects that the channel has been idle for a certain time interval, it choose a random backoff time within a contention window (CW). Frame transmission can start only if the STA detects an idle channel for this additional random amount of time.

EDCA introduces differentiated channel access probabilities to frames contending for accessing the channel. Four access categories (AC) are implemented at each quality of service station (QSTA) to prioritize the frames. Prioritization of different traffics is realized by mapping frames to the proper $\mathrm{AC}$ according to their QoS requirements and by assigning to each AC an appropriate set of four EDCA parameters. These parameters are used for regulating the channel contention phase and they are periodically broadcasted by the QAP through the beacon frames. The common idea of EDCA is to minimize packet transmission collision. However when the traffic load increases, without flow admission control, collision rate cannot be significantly reduced by only adjusting the backoff interval. i.e the collision rate increased with the increase of the traffic load. In this paper we propose a differentiated service approach for enhancing the QoS with differing requirement levels of service by admission control in the MAC layer of the QoS access point (QAP). The remaining part of the paper is organized as follows. In section II we discuss the IEEE 802.11e MAC layer QoS Mechanism section III priority scheduling for QAP section IV the simulation and analysis. Finally in section $\mathrm{V}$ conclusions are drawn.

\section{IEEE 802.11E MAC LAYER QOS MECHANISM}

The IEEE working group initiated the IEEE 802.11e to support for QoS including real time applications. The IEEE 802.11e standard provides the QoS support through enhancement of MAC sub-layer. It enables an access point (AP) to schedule resources based on STA data rate and latency needs, improve the wireless bandwidth efficiency and packet overheads and reduces latency by prioritizing wireless packets based on traffic types. A STA that supports IEEE 802.11e QoS enhancement mechanism is referred to as a QoS enhancement station (QSTA). When an AP that support these mechanism is referred to as a QoS enhancement AP (QAP). The channel access method introduced by IEE $802.11 \mathrm{e}$ is HCF, is combination of DCF and PCF $[1,3,4]$. HCF has two modes of operations; a contention based EDCA and a controlled channel access method, HCCA.

The four AC's of IEEE 802.11e is labeled as Voice ( $\left.A C_{-} V O\right)$, Video $\left(A C_{-} V I\right)$, Best Effort $\left(A C_{-} B E\right)$ and Background $\left(A C_{-} B K\right)$ as in fig. 1. These AC's are considered as four separate queues each with their own MAC CSMA/CA mechanism. The priority mechanisms used for accessing the channel are Arbitration Inter frame space Number (AIFSN) and contention window back-off intervals $\left(C W_{\min }\right.$ and $\left.C W_{\max }\right)$. Each AC contends independently for access to the channel based on the above parameters within the QSTA. To avoid the collisions within the TC each AC independently starts counting an additional random number of time slots, known as a $C W$, before attempting to transmit data. The frames with highest priority will access the wireless channel than the one with other priority levels. Video and voice are the highest priority queues, BE is medium priority queue and $\mathrm{BK}$ is the lowest priority queue. Once the channel is accessed by a QSTA, it is allowed to hold the 
channel for a certain period known as transmission opportunity $(T X O P)$. From the above observation it is comprehensible that by tuning AIFSN, $C W_{\min }, C W_{\max }$ and TXOP limit it is possible to introduce a relative prioritization between the queues in winning access to the medium and transmitting the packets.

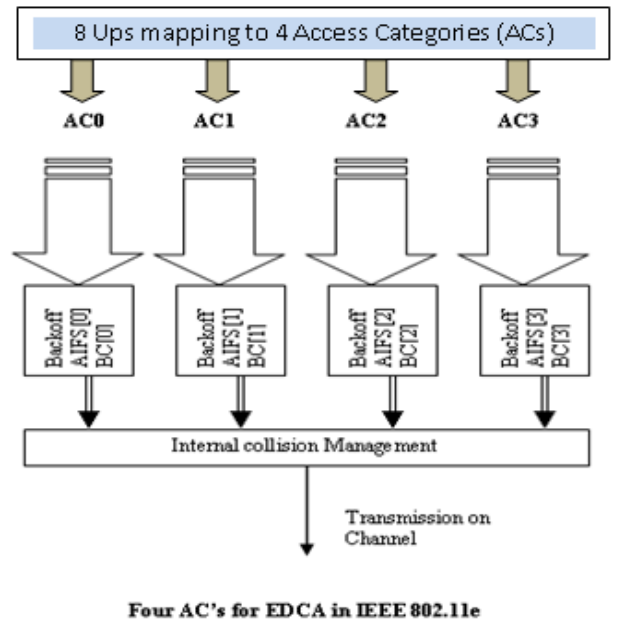

Fig.1. Four AC's in IEEE 802.11e.

Buffer over flow is one of the processes in most of the wireless devices MAC systems. Before the STA starts the transmission its MAC buffer holds the frame and waiting for the TXOP. As long as a STA wins the TXOP its MAC buffer remains empty and buffer never fill to exceed its capacity and hence packets are never lost. If a STA does not win enough TXOP, then the data frames are scheduled based on the priority. Hence when many STAs are contending to access the wireless medium, the frames assigned with highest priority in the queue gains the TXOP.

\section{PRIORITY SCHEDULING FOR QAP}

The priority scheme for differentiating the priority levels for the real time data frames in QAP is by managing the $C W$ adaptively. In this approach a new model is added in the MAC layer to manage the QoS. It differentiates the frames and put them in appropriate queues. The basic levels of end-to-end QoS can be provided across a heterogeneous network is as shown in the fig. 2. It represents three levels of end-to-end QoS[2, 6] as; Guaranteed Service (GS), Differentiated Service (DS) and Best Effort service (BE).

Guaranteed Service (GS): It is also known as hard QoS. Here there is an absolute resource reservation of network resources for specific traffic.

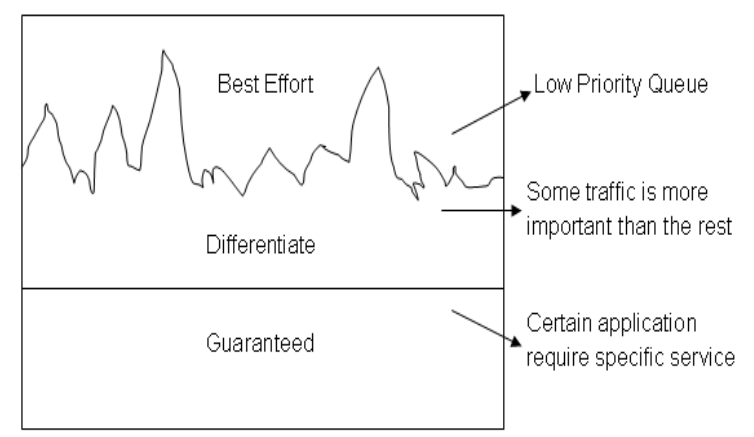

Fig.2. Three levels of End-to-End QoS
Differentiated Service (DS): It is also known as soft QoS. Some traffic is treated better than the rest. This is a statistical preference not a hard and fast guarantee. This is provided by classification of the traffic as priority queuing (PQ), custom queuing (CQ), weighted fair queuing (WFQ) and class based waited fair queuing (CBWFQ).

Best Effort (BE) Service: Also known as lack of QoS. BE service is basic connectivity with no guarantees. This is best characterized by FIFO, which have no differentiation between the frames.

The basic WLAN QoS architecture [2] introduces three fundamental pieces for QoS implementations;

- QoS related policy management and accounting functions to control administrator end-to-end traffic across a network in the server.

- QoS signaling between the QAP and QSTA.

- $\mathrm{QoS}$ in AP - queuing, scheduling, aggregation etc.

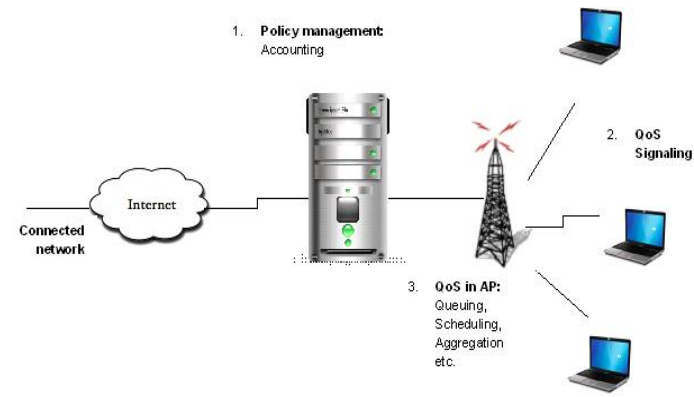

Fig.3. WLAN QoS network architecture

The enhancement in the MAC layer of the AP with the proposed priority scheduling techniques is as shown in fig.4.

Adaptive priority queuing is the simplest method providing service differentiation in a WLAN [2, 6]. In which each class of packets enters a separate buffer that is granted a specific priority and the packets in the high priority buffer are served before those of all lower priorities. In priority scheduling each packet is placed in one of the three queues based on the assigned priority. During transmission the algorithm gives high priority queues absolute preferential treatment over low priority queues. More importantly it is implemented in hardware and therefore can be applied to high speed infrastructure in both MAC and PHY layers of the network [5]. The basic architecture discussed above of that combines high priority queue and queue with adaptive priority allocation. This is called adaptive priority scheduling (APS).

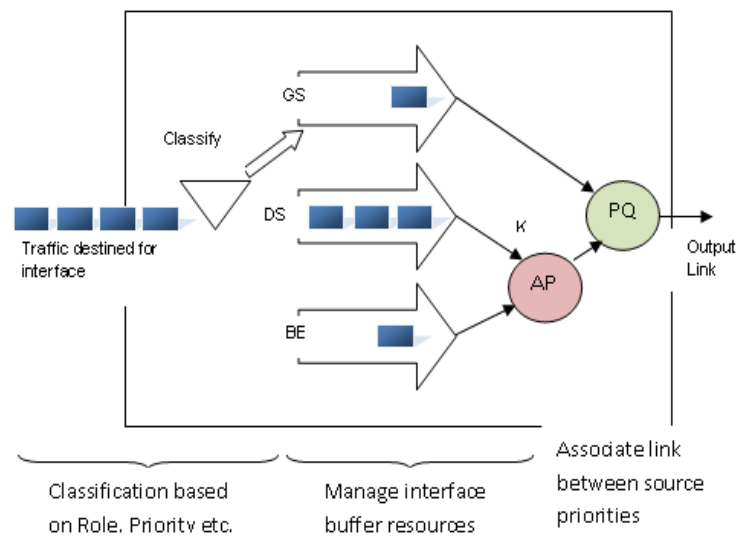

Fig.4. Service differentiation using three levels of queue 
Whenever a packet arrives to an AP the QoS management (QoSM) differentiates the real time application packets it is to be delivered without any delay and general packets. The real time traffic is put in to quality queue (Qq either GS or DS) and the general traffic in the Best Effort t Queue (BEq). The QAP contains two modules Quality Evaluation Module (QEM) and Priority Queue (PQ). QEM provide the required service differentiation by differentiating the real time application traffic and general frames, to the high priority quality queue such as GS, DS BE queues. After getting the packet type from QEM it does the queue assignment by the help of PQ module, if the queue is not full for both the type of packets. The packet forwarding in the three priority queue is done in a strict priority policy.

The basic QoS implementation has three main components.

In fig. 4 the Guaranteed Service traffic is put into the highest priority queue and has strict priority over the other two classes in a non preemptive way. The frames for this queue are selected based on the roles of the users and type of the traffic. This includes more time sensitive frames like confidential information from top brace employees in the organization, or network management packets; which demands express service without queuing delay or packet loss.

The differentiated Service is typically a real time applications usually has a quite strict QoS requirement on delay and should be given a guaranteed amount of bandwidth by adaptively reallocating the priority parameters. In reality the DS classtypically real time applications, usually have a quite strict QoS, requirements on delay and should be given a guaranteed amount of bandwidth [8]. In this queue there is a large amount of traffic or may be some ill behaved flows in the network without differentiation, real time traffic could suffer starvation.

In our priority scheduling technique we restrict our attention to the problem of the response time of the DS class under the worst case scenario. The frames in GS class are generally insensitive to delay, analysis for this scheme is not necessary.

\section{Priority Scheduling Algorithm:}

$$
\begin{aligned}
& f_{i}=i^{\text {th }} \text { frame inTransmission } \\
& f_{t}=\text { frame Type } \\
& G_{S}=\text { Guaranteed Service } \\
& G_{S Q}=G_{S} \text { Queue } \\
& P Q=\text { Priority Queue } \\
& D_{S}=\text { Differentiated Service } \\
& D_{S Q}=D_{\text {S }} \text { Queue } \\
& B E_{Q}=\text { BE Queue }
\end{aligned}
$$

$$
\begin{array}{cc}
\text { 1: } & \text { Receive } f_{i} \\
2: & f_{t}=Q E M\left(f_{i}\right) \\
3: & \text { If } f_{i}=G_{S} \\
4: & \text { If } G_{S Q}=\text { Full } \\
\text { 5: } & \text { Then drop } f_{i} \\
6: & \text { Else PQ }\left(f_{i}, G_{S Q}\right) \\
7: & \text { Else If } f_{t}=D_{S} \\
\text { 8: } & \text { If } D_{S Q}=\text { Full } \\
\text { 9: } & \text { Then drop } f_{i} \\
\text { 10: } & \text { Else PQ }\left(f_{i}, D_{S Q}\right) \\
\text { 11: } & \text { Else PQ }\left(f_{i}, B E_{Q}\right) \\
\text { 12: } & \text { End If }
\end{array}
$$

\section{2: End If}

\section{Performance Evaluation of the system}

$P_{d}$ is the propagation delay time taken to transmit between source to AP and AP to the QSTA, in addition with queuing delay $T$. A transmission cycle of DCF consists of DIFS deferral, backoff, data, transmission, SIFS deferral and ACK transmission.

Average Back-off Time is $B T_{\text {avg }}=\frac{C W_{\min } \times T \text { slot }}{2}$

Data transmission delay;

$T_{D-\text { data }}=T_{P H Y}+T_{H-\text { data }}+T_{\text {data }}$

ACK transformation delay; $T_{D-a c k}=T_{P H Y}+T_{A C K}$

The maximum throughput $T_{\max }$ of the system is given as;

$$
T_{\max }=\frac{L_{\text {data }} \times 8}{T_{D-\text { data }}+T_{D-A C K}+2 P_{d}+T_{\text {DIFS }}+T_{\text {SIFS }}+B T_{\text {avg }}}
$$

Where, $L_{\text {data }} \times 8$ bits are the data packet size. Packet delay is the time elapsed between the transmission of a packet and its successful reception. The minimum delay $D_{\min }$ of the system is given as, $D_{\text {min }}=T_{D-\text { data }}+P_{d}+T_{D I F S}+B T_{a v g}$ The performance of $D_{\min }$ and $T h_{\max }$

\section{SIMULATION AND ANALYSIS}

The performance of MAC layer with enhanced QoS by service differentiation (QSD) is simulated using NS-2. The QSD system is tested for real time (RT) data frames. The simulation parameters are as in Table 1; 
Table: 1 Simulation parameters

\begin{tabular}{|l|l|}
\hline Parameters & Values \\
\hline MAC Header & 34 bytes \\
\hline PHY Header & 16 bytes \\
\hline ACK & 14 Bytes \\
\hline RTS & 20 bytes \\
\hline CTS & 14 Bytes \\
\hline Slot Time & 50 Micro Sec. \\
\hline SIFS & 28 Micro Sec. \\
\hline DIFS & 128 micro sec \\
\hline CWmin & 31 \\
\hline CWmax & 1023 \\
\hline
\end{tabular}

The different types of traffics used for simulation are streaming video and FTP/TCP. Where the queue can accommodate a maximum up to 50 packets and rest of the packets are truncated by drop tail mechanism.

The QAP and QSTA are shown in the network architecture can able to handle data rate of 2Mbps. The QSTA can generate and receive a RT data having packet size 1500 bytes (FTP data). The other STA's can generate and receive the TCP/FTP packets with a constant bit rate (CBR) having packet sixe 1460bytes. The data from the Internet and through the server is access by the QSTA's. In the AP the QoS algorithm (QEM) processes this to accommodate the frame in GS, DS and BE queue. Once the connection is established with the server it can send the data through DS queue.

The performance of this mechanism can be analyzed for throughput and delay of QSD in comparison to legacy MAC for RT streaming data. On progress of transmission delay is added to the TCP/FTP data packets. Comparing QSD and legacy MAC the delay analysis is shown in fig.5.

The delay is comparatively lower than the legacy MAC. Throughput analysis is described between QSD and legacy MAC is shown in fig.6. In QSD as delay decreases the throughput increases for RT like streaming video frames. The overall throughput with the presence of GS, DS and BE traffic using QSD and legacy MAC is calculated by considering both type of flows simultaneously. This remains same as in fig. 5 and fig.6. This is because the scheme just provide service to real time traffic by adding delay to best effort traffic it gain throughput for the real time traffic, without any guaranteed service to BE traffic.

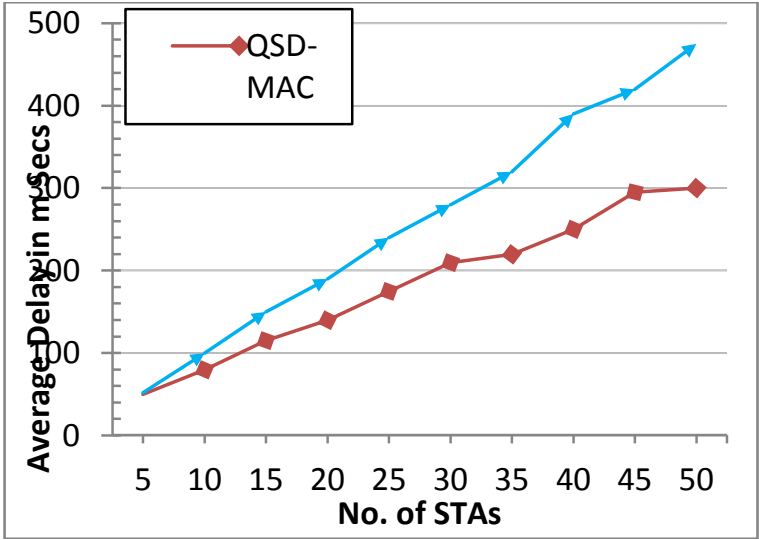

Fig. 5 Comparison of Average Delay

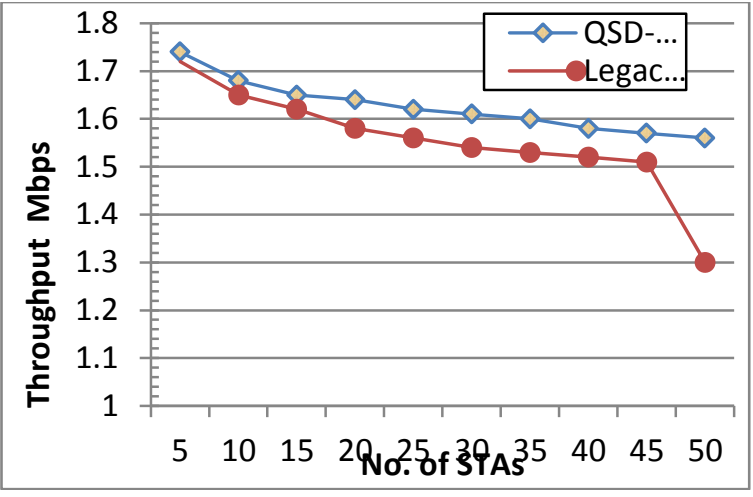

Fig.6. Comparison of Throughput Analysis

\section{CONCLUSION}

The AQoS mechanism using service differentiation (QSD) demonstrates the performance of real time traffic is enhanced significantly. The simulation result for RT streaming video and FTP traffic shows that it gains better throughput for RT traffic. The overall throughput of the system remains same as the legacy MAC because the scheme provides service to only the real time traffic (DS) without considering GS and BE Queues.

\section{ACKNOWLEDGMENTS}

The authors would like to thank the Vice-Chancellor of SASTRA University for his support.

\section{REFERENCES}

[1]. A. Banchs and X. Perez, .Providing throughput guarantees in IEEE 802.11 wireless LAN,. Proc. IEEE Wireless Communications and Networking Conference, 2002 (WCNC2002)., pp. 130.138.

[2]. S. Blake, D. Black, M. Carlson, E. Davies, Z. Wang, and W. Weiss, "An architecture for differentiated services," $R F C$ 2475 Internet Engineering Task Force, Dec. 1998.

[3] .IEEE Standard for Information technologyPart11: Amendment: Medium Access Control (MAC) Quality of Service (QoS) Enhancements, 2003rd ed., IEEE P802.11e/D13.0, Draft Supplementto IEEE STD 802.11 1999, 2005.

[4]. IEEE Std. 802.11-1999, "Part11:Wireless LAN Medium Access Control (MAC) and Physical Layer (PHY) Specifications", Reference number ISO/IEC 880211:1999(E), IEEE Std 802.11, 1999 edition IEEE (1999). Revised in (2003).

[5]. IEEE 802.11e, "Supplement to Part 11: Wireless Medium Access Control (MAC) and physical layer (PHY) specifications: Medium Access Control (MAC) Enhancements for Quality of Service (QoS)", IEEE Std. 802.11e-2005 IEEE (2005).

[6]. K. Nichols, V. Jacobson, and L. Zhang, "A two-bit differentiated services architecture for the Internet," $R F C$ 2638, Internet Engineering Task Force, July 1999.

[7]. Part 11: Wireless LAN Medium Access Control (MAC) and Physical layer (PHY) specifications: Amendement 7: Medium Access Control (MAC) Quality of Service (QoS) Enhancements, IEEE Unapproved Draft, Rev. D12.0, 2004.

[8]. Rathnakar Acharya, Dr. V. Vityanathan, Dr. Pethur Raj Chellaih WLAN QoS Issues and IEEE 802.11e QoS Enhancement International Journal of Computer Technology and Engineering 2010 Vol.2(1): 143-149 ISSN: 1793-821X. 